\title{
BMJ Open Lived experience of dietary change among Chinese colorectal cancer survivors in Hong Kong: A qualitative study
}

\author{
Janet Yuen Ha Wong (D) , ${ }^{1}$ Judy Wai Chu Ho, ${ }^{2}$ Antoinette Marie Lee, ${ }^{3}$ \\ Daniel Yee Tak Fong (D) , ${ }^{1}$ Natural Chu, ${ }^{2}$ Sharron Leung, ${ }^{4} Y$ Y Wynnie Chan, ${ }^{5}$ \\ Sharon HS Lam, ${ }^{2}$ Ivy P F Leung, ${ }^{6}$ Duncan Macfarlane, ${ }^{7}$ Ester Cerin, ${ }^{8}$ Aliki J Taylor, ${ }^{9}$ \\ Kar Keung Cheng ${ }^{9}$
}

To cite: Wong JYH, Ho JWC, Lee AM, et al. Lived experience of dietary change among Chinese colorectal cancer survivors in Hong Kong: A qualitative study. BMJ Open 2021;11:e051052. doi:10.1136/ bmjopen-2021-051052

- Prepublication history for this paper is available online. To view these files, please visit the journal online (http://dx.doi org/10.1136/bmjopen-2021051052).

Received 10 March 2021 Accepted 11 August 2021
Check for updates

(C) Author(s) (or their employer(s)) 2021. Re-use permitted under CC BY-NC. No commercial re-use. See rights and permissions. Published by BMJ.

For numbered affiliations see end of article.

Correspondence to Dr Daniel Yee Tak Fong; dytfong@hku.hk

\section{ABSTRACT}

Objectives This is a qualitative study which aims to understand the lived experience of dietary changes among Chinese survivors of colorectal cancer who participated in a dietary intervention.

Setting The surgical and oncological departments of four public hospitals in Hong Kong.

Participants Fifty-five Chinese colorectal cancer survivors who were aged 18 years or above and had received potentially curative treatment in the surgical and oncological departments in Hong Kong were examined. Participants' mean age was 64 years, with 29 (53\%) males.

Intervention A 12-month dietary intervention delivered via face-to-face motivational interviews, fortnightly motivational phone calls, monthly electronic pamphlets, quarterly newsletters and quarterly group meeting. Outcome measure We adopted the qualitative approach to capture participants' perspectives and to apply the understanding pragmatically in everyday life. Content analysis was conducted.

Results We identified themes of motives to changes of dietary practices including (1) individual commitment to dietary change; (2) adaptive strategies in interpersonal contexts and (3) working with healthcare professionals during the journey.

Conclusions The findings demonstrated how Chinese custom and culture posing unique challenges to colorectal cancer survivors and the need of having dietary advice from healthcare professionals. Participants were motivated to change their eating habits by support from family, friends and healthcare professionals. Our findings could help healthcare professionals provide specific dietary advice and guidance to Chinese colorectal cancer survivors.

Trial registration number NCT01708824.

\section{INTRODUCTION}

Colorectal cancer (CRC) is the most common cancer in Hong Kong. ${ }^{1}$ Although surgical resection and adjuvant therapy are curative treatments for CRC, the recurrence rate was

\section{Strengths and limitations of this study}

- To the best of our knowledge, this is the first study to investigate the lived experience of dietary change according to a prescribed dietary intervention among Chinese colorectal cancer (CRC) survivors

- This study used qualitative approach with content analysis. During the process of data collection, reflexivity and the trust among the participants and research assistants and the participants was conducted to enhance the rigour of the study.

- Although we have collected interview data until data saturation, all study participants had joined our behavioural intervention programme, the experience they shared may not be transferable to all CRC Chinese survivors.

still $40 \%-60 \%$ in the first 3 years after treatment. ${ }^{2}$ The latest data revealed convincing evidence that inadequate dietary fibre and high red and processed meat intakes increase the risk of CRC. ${ }^{3}$ In addition, some observational studies also found that diet associated with CRC recurrence rates ${ }^{4}$ and post-treatment cancer outcomes. ${ }^{5}$ For cancer survivors, there were little evidence about cancer diagnosis ${ }^{6}$ and fear of cancer recurrence ${ }^{7}$ as motives for positive lifestyle changes. While the evidence that healthier styles including dietary change is growing, it is crucial to understand cancer survivors' perspectives on dietary changes, so as to translate the scientific knowledge to evidence-informed health promotion.

In many cultures, food serves as nutrition and social functions. ${ }^{8}$ In Chinese culture, food dishes are served communally and shared on a round table to establish and enhance interpersonal relationships. This contrasts with Western culture, wherein food is often served individually, without sharing. ${ }^{8}$ We anticipated 
that Chinese cancer survivors would face many challenges in adopting dietary changes. An in-depth understanding of their beliefs, attitudes and choices is needed to inform future interventions targeting dietary management for CRC survivors in Chinese settings. In 21st century, clinical practice and other healthcare services have increasingly been affected by cultural diversities. As Chinese are the world's largest ethnic group, healthcare professionals' culture competence to deliver high-quality patientcentred practice towards Chinese patients is a necessary professional skill. ${ }^{9}$

\section{PURPOSE}

To understand the lived experience of dietary changes among Chinese CRC survivors after participating in a behavioural intervention program targeting diet and physical activity.

\section{METHODS}

\section{Design}

This study presents the individual and group interview data from a large, multicentre, randomised controlled trial (RCT) that evaluated two 12-month behavioural interventions in a programme named 'Moving Bright, Eating Smart' for improving CRC survivors' dietary habit and physical activity level. Participants were randomised into intervention groups (physical activity intervention, dietary intervention or both interventions) or control groups. The intervention was delivered by using Health Action Process Approach (HAPA) ${ }^{10}$ and the content was developed based on the theory of planned behaviour. ${ }^{11}$ The intervention consisted of two face-to-face motivational interviews, fortnightly motivational phone calls, monthly electronic pamphlets, quarterly newsletters and quarterly group meeting, with the aim of achieving less than five servings of red/processed meat weekly and two servings of refined grains daily. Participants received the interventions via face-to-face motivational interviews, phone calls, educational pamphlets, newsletters and group meetings to facilitate their changes towards preset behavioural targets. Details of the trial protocol have been published. ${ }^{12}$

This qualitative study was conducted with focus on capturing social and cultural perspectives held by subgroups of people and applying the resulting understandings pragmatically to everyday life. ${ }^{11}$ We emphasise discovering how participants integrate their personal knowledge, health beliefs and practices into their lives and identifying the culturally embedded values that interact with practices. ${ }^{13}$ These emphases corresponded with our aim of thoroughly understanding how Chinese cultural background and lifestyle may influence CRC survivors' experiences of dietary changes from their own perspectives.

\section{Sample}

Participants were recruited from the surgical and oncological departments of four public hospitals in Hong
Kong that treat patients with CRC. Inclusion criteria for participation were as follows: (1) histologically proven colorectal adenocarcinoma; (2) previously received potentially curative treatment; (3) aged 18 years or above and (4) able to give informed consent. Participants, who had persistent or recurrent disease, were receiving cancer treatment, had hereditary CRC syndrome(s), had difficulties in receiving the proposed interventions or were unable to read Chinese were excluded.

A total of 229 participants were recruited to join the 'Moving Bright, Eating Smart' programme and evaluated via an RCT. All 100 whom completed the 12-month diet intervention were invited for the interviews, and 55 attended the interviews in the present study, yielding a response rate of $55 \%$. We ended the participant recruitment after completion of interviews with 55 participants because there was no new relevant knowledge obtained. All participants received a written participant information sheet explaining the study. Written consent prior to the interviews was obtained.

\section{Data collection}

Two female research assistants who had experience in conducting qualitative interviews were trained for consent procedure, information confidentiality, questioning techniques based on the interview guide, interview probing, sensitivity, clarifying and appropriate reassurance. They conducted 14 individual interviews and 16 group interviews comprising less than six persons using an interview guide. Box 1 shows the questions of the interview guide. Taking up a reflexive approach, our questions were developed from our clinical experiences in working with patients with CRC. All interviews were conducted in a private room in a hospital. Duration of the either individual or focus group interviews ranged from $35 \mathrm{~min}$ to 1 hour and $10 \mathrm{~min}$. All interviews were audiotaped and transcribed verbatim in Chinese in order to preserve their linguistic authenticity.

During the process of data collection, reflexivity and the trust among the research assistants and the participants was emphasised. ${ }^{14}$ It is a process of attending systematically to the context of knowledge construction in the researchers or research assistants in this study. Our research team consists of practising colorectal surgeon, nurses and researchers who have been working on health

\section{Box 1 Interview guide}

- Overall, how do you feel about the programme 'Moving Bright, Eating Smart'? Which is the most valuable part in this programme? Do you have any difficulties in following the dietary intervention of the programme?

What are the factors that sustained your dietary changes?

- What are the reasons that motivated you to join this programme? What are the barriers you faced in following the programme?

- Do you have any recommendations for us to improve the programme? Will you recommend this programme to other cancer survivors? 


\begin{tabular}{lc}
\hline Table $1 \quad$ Participant characteristics $(\mathrm{n}=55)$ & $\begin{array}{r}\text { No }(\%) \text { or } \\
\text { mean } \pm \text { SD }\end{array}$ \\
\hline $\begin{array}{l}\text { Gender } \\
\text { Male }\end{array}$ & $29(52.7)$ \\
\hline Female & $26(47.3)$ \\
\hline Age & $64.05 \pm 9.98$ \\
Cancer site & \\
\hline Colon & $36(65.5)$ \\
\hline Rectum & $19(34.5)$ \\
AJCC cancer staging & \\
\hline I & $12(21.8)$ \\
\hline II & $24(43.6)$ \\
\hline III & $18(32.7)$ \\
\hline IV & $0(0.0)$ \\
\hline Unknown & $1(1.8)$ \\
\hline Type of treatment received & \\
\hline Surgery & $55(100)$ \\
\hline Chemotherapy & $32(58.2)$ \\
\hline Radiotherapy & $7(12.7)$ \\
\hline Stoma status & $51(92.7)$ \\
\hline No stoma & $0(0.0)$ \\
\hline Temporary & $4(7.3)$ \\
\hline Permanent & \\
\hline AJCC The American Joint Commitee & \\
\hline
\end{tabular}

AJCC, The American Joint Committee on Cancer.

and quality-of-life research. The research assistants were trained in questioning techniques by experienced researchers in the team. They were obliged to interact with the participants being studied..$^{15}$ The research assistants established relationships with participants in quarterly group meetings and used talk-in-interaction, whereby they reflexively constructed interactions as they discussed practical issues with the participants. ${ }^{16}$ Additionally, whenever new topics emerged in the interviews, further enquiries were made to facilitate further knowledge production. Reflexivity during data collection promoted rigour in this study. ${ }^{17}$

\section{Data analysis}

Content analysis was conducted. ${ }^{18}$ The data were grouped into broad topics before the contents relevant to diet were extracted by two independent doctoral-prepared nursing researchers (SL and JYHW) by using inductive approach. Then, the data were grouped according to a series of categories. The construction of categories was done according to repeated revisions and manifestation of latent coding. ${ }^{19}$ Themes were condensed into theoretical constructs by observing the typological patterns and relevance in the data. Reflections were also done to look for the motivation of diet change and actual behaviours of diet change of each participant to ensure the themes were sensible and structural. We also involved social interpretation of the results in local context and specific situations. Our own afterwards, a two-stage process was repeated by a second coder (JYHW) to ensure accuracy and consistency of the categories and themes. ${ }^{20}$ The second coder generated own codes and categories independently by reading the verbatim transcripts. Then, the first four authors (JYHW, JWH, AML and DYTF) and research assistants discussed and verified the codes, categories and themes based on our prior assumptions about patients with CRC in managing their health and how these codes, categories and themes made sense in the conceptualisations at several consensus meetings. Member checking was conducted informally in the quarterly group meetings.

\section{Patient and public involvement}

This study is initiated from the colorectal and oncology teams in four public hospitals in Hong Kong. Patients, caregivers and public have not involved in the research design, planning, implementation or reporting of our research.

\section{RESULTS}

\section{Participant characteristics}

Table 1 shows participants' characteristics. Over half of the participants were male $(\mathrm{n}=29,52.7 \%)$. Participants' mean age was 64.05 years ( $\mathrm{SD}=9.98$ years). The overarching theme of this study was the motives to change dietary practices. Table 2 shows the corresponding three broad themes, including: (1) individual commitment to dietary change; (2) adaptive strategies in interpersonal contexts and (3) working with healthcare professionals during the journey.

\section{Individual commitment to dietary change}

Maintaining a healthy lifestyle is a lifelong commitment. Our participants revealed the major difficulties and societal barriers they encountered in Chinese context and their individual corresponding strategies for dietary change. This theme was divided into two categories:

Table 2 The themes and categories identified in this study

\begin{tabular}{|c|c|}
\hline Broad themes & Categories \\
\hline $\begin{array}{l}\text { Individual } \\
\text { commitment to } \\
\text { dietary change }\end{array}$ & $\begin{array}{l}\text { Barriers to dietary change. } \\
\text { Facilitators for dietary change. }\end{array}$ \\
\hline $\begin{array}{l}\text { Adaptive strategies } \\
\text { in interpersonal } \\
\text { contexts }\end{array}$ & $\begin{array}{l}\text { Avoid feeling isolated while eating } \\
\text { with others. } \\
\text { Challenges during Chinese festivals } \\
\text { and innovative strategies to } \\
\text { overcome these challenges. }\end{array}$ \\
\hline $\begin{array}{l}\text { Working with } \\
\text { healthcare } \\
\text { professionals during } \\
\text { the journey }\end{array}$ & $\mathrm{N} / \mathrm{A}$ \\
\hline
\end{tabular}

N/A, not available. 
'barriers to dietary change' and 'facilitators for dietary change'.

\section{Barriers to dietary change}

Traditional Chinese cultural beliefs and practices create difficulties in compliance with dietary changes. A typical example is the belief that rice and meat are the main sources of energy and nutrition among Chinese people. Our participants expressed difficulties in adjusting their accustomed values and habits. Nevertheless, the participant changed his diet with reduced portion of rice.

I didn't think of avoiding rice. Chinese people have eaten rice for thousands of years.... Therefore, to be honest, I sometimes eat one to two spoons of rice. (Male, aged 57)

In the past, we grew up by eating rice. (Female, aged 52).

Of course, there were difficulties. We are Chinese, who always like to eat meat. How can I ask my family to avoid meat? But I tried my best to eat less. (Male, aged 59)

\section{Facilitators for dietary change}

Some Chinese beliefs contributed to the participants' perceptions of changing diet and adopting a healthy diet.

Western doctors usually don't ask you to avoid eating anything. Traditional Chinese medicine practitioners always ask you to avoid eating this and that. (Male, aged 47)

Some participants highlighted their awareness of the importance of healthy diet after treatment because of the severity of their disease and the possibility of recurrence.

Sometimes, I have no idea about eating wrongly. After learning what to eat and what to avoid for colorectal cancer, now I know. (Male, aged 51)

Many participants found that the suggested dietary changes had additional benefits. They experienced improvement in their general well-being. These participants successfully developed an interest in maintaining a healthy diet through selection of better food and even making their own food.

The bread we can buy out there is usually made with plenty of butter, emulsifier, and MSG [monosodium glutamate]. I wanted to have healthier food and so I started making my own bread. Most importantly, they don't create problems like dry mouth and headache. (Female, aged 42)

\section{Adaptive strategies in interpersonal contexts}

Eating is a social event. Eating together with family members and friends builds relationships and helps people feel more socially connected. It can be a challenge for the host when one of the guests has many specific dietary restrictions.
Avoid feeling isolated while eating with others

Traditional Chinese dinners focus on sharing. Participants expressed their wish to enjoy food together with their friends and relatives. Unfortunately, they found that the food most welcomed by others was usually unsuitable for them. Although there was a dilemma between staying on a healthy diet and maintaining harmony with others, the participants found some strategies in balancing both sides.

When I eat with friends, I don't want to give the impression that I am difficult to get along with. I will just give up and eat a small portion of meat if they invite me repeatedly. (Male, aged 67)

Fish is healthy because it is white meat. My family supports me to eat more fish, but they don't want to sacrifice and change their own diet. Sometimes they prefer pork. Therefore, I have to strike balance by preparing separated dishes. (Female, aged 56)

\section{Challenges during Chinese festivals and innovative strategies to} overcome these challenges

Participants were exposed to many traditionally meaningful but high-carbohydrate foods and had difficulties resisting them. A participant gave allowance to herself in the Chinese New Year while another participant developed innovative method to tackle the dilemma.

It is inevitable that I tend to eat more during Chinese New Year. It is impolite to reject the snacks and alcohol prepared by relatives and friends. (Female, aged 65)

Recognising the possible detrimental health impact of the traditional festival foods, some participants developed innovative strategies to overcome these difficulties. For example, some made their own healthy version of rice dumplings in Dragon Boat Festival.

I added more green beans to replace some glutinous rice in making my dumplings. (Male, aged 79)

\section{Working with healthcare professionals during the journey}

The information provided from healthcare professionals were evidence based. In addition to health information, healthcare professionals also provided emotional support. Participants' confidence and determination were noted in the interviews. This reassurance was found to be effective in helping participants overcome fear and doubt.

Some friends and relatives had cancer despite maintaining healthy diets all along, while some didn't get cancer even they had bad diets for years. Their information made me feel lost. After I joined the program, I became more focused. There are lots of helpful professionals around. The information I got was scientifically sound and logical. Now I can hold firm to my belief and I will follow it no matter what happens in the future... You guys are stand behind us. Following your suggested diet, I know what is good or what is 
bad. I personally think it is very useful. When you said that I completed the programme and I could take care of myself. I would say.... I will miss the programme... hahaha... (Female, aged 47)

Participants, after joining the programme, received useful health information from a variety of sources, including mailed pamphlets, emails and phone messages. Through these informative resources given by healthcare professionals, they gained knowledge about pursuing a healthy diet.

I used to think that red meat only referred to beef. But now I know that it also includes pork and lamb. Also, I once thought white rice was a plant so it must be healthy. But now I understand that it is processed. Red cargo rice is better since it is more natural. (Female, aged 52)

After listening to you guys, now, I know how to recognize whole grain food. (Female, aged 42)

\section{DISCUSSION}

\section{Summary}

This is, to our knowledge, the first study to investigate the lived experience of dietary change according to a prescribed dietary intervention among Chinese CRC survivors. This study presented unique challenges relevant to Chinese culture and lifestyle, and it reported how the cancer survivors coped with these challenges.

From our findings, we understand that Chinese custom and culture pose unique challenges to CRC survivors. One example is the belief that rice and fatty meat are the main sources of energy among Chinese people. We attribute this belief to the strong agricultural background of traditional Chinese culture, which is different from Western countries. In the USA, consuming red meats are viewed as necessary and satisfying. ${ }^{21}$ Historically, American and European luxury shops were the first to stock canned meat. ${ }^{22}$ Therefore, meats are also viewed as luxuries and providing a sense of pride. However, Chinese people, as the Chinese saying that 'Min Yi Shi Wei Tian' (Food is the first necessity for the people), view rice and fatty meat as primary need, which may make the dietary change more difficult.

Traditional Chinese cultural beliefs and practices of eating as sharing is significant in the value formation in a family. Specifically, dishes sharing and eating together is an expression of the degree of interpersonal relationship. ${ }^{8}$ Another previous qualitative study in patients with heart failure found consistent findings that sharing food is a typical custom in China and the dietary changes might create a burden for family members. ${ }^{23}$ Similarly, their study demonstrated the patients' struggles in interpersonal relationships during dietary change. The struggles would be intensified during festivals. Our participants particularly mentioned Chinese New Year and Dragon Boat Festival, in which people shared traditional and popular foods with family and friends. Those cultural perspectives on dietary change challenges have provided as more understanding of how culture affects their responses to the illness and are very helpful for healthcare professionals in customising the healthcare plan.

Although participants faced many challenges, they developed innovative strategies to cope with the challenges, for examples, reducing the portion of rice and fatty meat, having separated dishes and having some replacements for unhealthy food. In addition, many participants indicated that they saw their own lack of awareness and improper food intake as contributing to their cancer. Descriptions of vulnerability and self-blame among cancer survivors can be found consistently throughout the literature, not only in Chinese populations but also Chinese immigrant communities. ${ }^{24}{ }^{25}$ In Buddhist philosophy, Chinese patients tend to have self-blaming responses towards cancer and regard the cause of cancer as sins. ${ }^{26}$ Nevertheless, some survivors were active and committed to dietary changes with support and guidance. The adherence to treatment behaviours can be explained by the reactions of gaining back a sense of control in a powerless sick condition. ${ }^{27}$ All the above findings provide a deepened understanding on how Chinese culture drives CRC survivors' behaviours and highlight the importance of designing interventions that are aligned with culturally compatible beliefs.

Our findings confirmed the need for dietary advice from healthcare professionals. The participants preferred scientific evidence and guidance from healthcare professionals to information from other resources. In addition, our healthcare professionals in this study are all Chinese who understand CRC survivors' cultural needs, so that they are able to play an important role of alleviating participants' fears and worries regarding the selection of food. Therefore, post-treatment programme should not only provide relevant nutritional information on dietary changes, but also address the need of CRC survivors for psychological support during their cancer journey. Moreover, the findings underscored that healthcare professionals should be well equipped with the cultural sensitivity and culture competence ability so that they could provide health advice to CRC survivors effectively.

The identified themes in this study were (1) individual commitment to dietary change; (2) adaptive strategies in interpersonal contexts and (3) working with healthcare professionals during the journey. It was supported by the HAPA, which integrated socialcognition theory, theory of reasoned action and volition theories. ${ }^{28}$ In HAPA, the dietary change can be explained by two stages. First, our CRC survivors had an intention and a commitment to change because of their cultural belief that diet was one of the key factors contributing to their diseases. Second, owing to the 12-month behavioural interventions provided to the CRC survivors and the healthcare professionals' support during the journey, they had the initiation to create adaptive strategies and had efficacy to maintain the dietary change. This was possible because of 
the stage-matched conditions, which was one of the major and unique principles in HAPA.

\section{Limitations}

There are several limitations in this study. First, the findings may not be transferable to all CRC survivors, because the study participants were recruited from a single region in Hong Kong and from a sample of people who had joined a behavioural intervention programme in local public hospitals. Second, the trained research assistants who collected the data were not conducted the data analysis but just participated in discussion of finding at the consensus meetings. Therefore, it might not be the optimal arrangement in demonstrating reflexivity. Third, social desirability bias might be possible, since participants had established relationships with the interviewers in quarterly group meetings. Therefore, they might tend to report positive aspects of the programme.

\section{Implications}

This study provided a better understanding of dietary changes among CRC survivors has potential import for reducing cancer recurrence, improving long-term survival and preventing secondary cancer development. It is recommended from our findings that scientific evidence should be incorporated in the dietary behavioural intervention, for examples, reminding for having less red meat, clarifying that rice is not only source of energy, emphasis on quantitative guidance, consideration on serving individual portions in family. Also, when healthcare professionals delivered this intervention, they should be mindful about the cultural factors and provide opportunities for patients to alleviate their fears and worries regarding the selection of food and support them in the dietary change journey. In addition, the government can promote social norm based messages with incorporation of Chinese cultural context for educating general public about healthy diet in the community.

\section{CONCLUSION}

The participants were found to be motivated in changing their eating habits by support from family, friends and healthcare professionals. Our findings support the HAPA and are highly relevant to the unique Chinese cultural context and provide insights to enable healthcare professionals to offer specific dietary advice and guidance to these survivors. Cultural competency is recommended to be strengthened in order to provide patient-centred care to CRC survivors.

\footnotetext{
Author affiliations

${ }^{1}$ School of Nursing, Li Ka Shing Faculty of Medicine, University of Hong Kong, Hong Kong, Hong Kong

${ }^{2}$ Division of Colorectal Surgery, Department of Surgery, Li Ka Shing Faculty of Medicine, University of Hong Kong, Hong Kong, Hong Kong

${ }^{3}$ Department of Psychiatry, Li Ka Shing Faculty of Medicine, University of Hong Kong, Hong Kong, Hong Kong

${ }^{4}$ Division of Nursing Education, Hong Kong Baptist University, Kowloon, Hong Kong
}

${ }^{5}$ School of Professional and Continuing Education, University of Hong Kong, Hong Kong, Hong Kong

${ }^{6}$ Department of Dietetics, Queen Elizabeth Hospital, Hospital Authority Kowloon Central Cluster, Kowloon, Hong Kong

${ }^{7}$ Institute of Human Performance, University of Hong Kong, Hong Kong, Hong Kong ${ }^{8}$ School of Public Health, Li Ka Shing Faculty of Medicine, University of Hong Kong, Hong Kong, Hong Kong

${ }^{9}$ Department of Public Health, Epidemiology and Biostatistics, University of Birmingham, Birmingham, UK

Contributors JYHW conducted the data analysis and drafted the manuscript. JWCH conceived of the study, participated in its overall design, obtained funding, coordinated the research team and drafted the manuscript. AML participated in the study design and conducted the data analysis. DYTF participated in the study design, determined the study sample size and method of statistical analysis, and supervised the randomisation process. NC collected the data. SL participated in the study design, data collection and data analysis. YYWC participated in the design of dietary intervention and assessment. SHSL participated in the design of dietary intervention and assessment. IPFL participated in the design of dietary intervention and assessment. DM and EC participated in the study design, advised on behavioural strategies to be adopted, and determined the content of physical activity intervention and its assessment. AJT conceived of the study and participated in the overall study design. KKC conceived of the study, obtained funding and supervised the overall study design. AML, DM, DYTF, SL, EC, YYWC, IPFL, SL, AJT and KKC revised the manuscript critically for important intellectual content. All authors read and approved the final manuscript.

Funding This study was partially supported by The World Cancer Research Fund International and the Wereld Kanker Onderzoek Fonds (WCRF NL). WCRF reference number: 2012/595.

Competing interests None declared.

Patient and public involvement Patients and/or the public were not involved in the design, or conduct, or reporting, or dissemination plans of this research.

Patient consent for publication Not required.

Ethics approval The study has been approved by the Institutional Review Boards of the Hong Kong West Cluster (UW 12-478), Hong Kong East Cluster (HKEC-2012068) and Kowloon West Cluster (KW/EX-13-002(59-02)) of the Hospital Authority in Hong Kong.

Provenance and peer review Not commissioned; externally peer reviewed.

Data availability statement No data are available. Data are available on request due to privacy or other restrictions. Requests for sharing study data must be made on specific grounds with the purpose of corroborating the study results in the interest of public health.

Open access This is an open access article distributed in accordance with the Creative Commons Attribution Non Commercial (CC BY-NC 4.0) license, which permits others to distribute, remix, adapt, build upon this work non-commercially, and license their derivative works on different terms, provided the original work is properly cited, appropriate credit is given, any changes made indicated, and the use is non-commercial. See: http://creativecommons.org/licenses/by-nc/4.0/.

\section{ORCID iDs}

Janet Yuen Ha Wong http://orcid.org/0000-0002-3000-4577

Daniel Yee Tak Fong http://orcid.org/0000-0001-7365-9146

\section{REFERENCES}

1 Hong Kong Cancer Registry. Overview of Hong Kong cancer statistics of 2015, 2017. Available: http://www3.ha.org.hk/cancereg/ pdf/overview/Summary\%20of\%20CanStat\%202015.pdf

2 Kanwar SS, Poolla A, Majumdar AP. Regulation of colon cancer recurrence and development of therapeutic strategies. World $J$ Gastrointest Pathophysiol 2012;3:1-9.

3 World Cancer Research Fund International. Cancer prevention \& survival: Summary of global evidence on diet, weight, physical activity \& what increases or decreases your risk of cancer. World Cancer Research Fund International, 2016. Available: https://www. wcrf.org/sites/default/files/CUP-Summary-Report-10Nov.pdf.

4 Cha R, Murray MJ, Thompson J, et al. Dietary patterns and information needs of colorectal cancer patients post-surgery in Auckland. N Z Med J 2012;125:38-46 http://www.nzma.org.nz/ 
journal/read-the-journal/all-issues/2010-2019/2012/vol-125-no-1356/ article-cha

5 Aune D, Chan DSM, Lau R, et al. Dietary fibre, whole grains, and risk of colorectal cancer: systematic review and dose-response metaanalysis of prospective studies. BMJ 2011;343:d6617.

6 Williams K, Steptoe A, Wardle J. Is a cancer diagnosis a trigger for health behaviour change? findings from a prospective, populationbased study. Br J Cancer 2013;108:2407-12.

7 Fisher A, Beeken RJ, Heinrich M, et al. Health behaviours and fear of cancer recurrence in 10969 colorectal cancer (CRC) patients. Psychooncology 2016;25:1434-40.

8 Ma G. Food, eating behavior, and culture in Chinese Society. J Ethn Foods 2015;2:195-9.

9 Mews C, Schuster S, Vajda C, et al. Cultural Competence and Globa Health: Perspectives for Medical Education - Position paper of the GMA Committee on Cultural Competence and Global Health. GMS J Med Educ 2018;35:1-17.

10 Schwarzer R, Luszczynska A. How to overcome healthcompromising behaviors - The health action process approach. Eur Psychol 2008;13:141-51.

11 Ajzen I. The theory of planned behavior. Organ Behav Hum Decis Process 1991;50:179-211.

12 Ho JWC, Lee AM, Macfarlane DJ, et al. Study protocol for "Moving Bright, Eating Smart"- A phase 2 clinical trial on the acceptability and feasibility of a diet and physical activity intervention to prevent recurrence in colorectal cancer survivors. BMC Public Health 2013;13:487.

13 Higginbottom GM, Pillary JJ, Boadu NY. Guidance on performing focused ethnographies with an emphasis on healthcare research. Qualitative Report 2013;18:1-16 https://nsuworks.nova.edu/tqr/ vol18/iss $9 / 1$

14 Roper JM, Shapira J. Ethnography in nursing research. Vol 1. Thousand Oaks, CA: SAGE, 2000.
15 Bikker AP, Atherton $\mathrm{H}$, Brant $\mathrm{H}$, et al. Conducting a team-based multi-sited focused ethnography in primary care. BMC Med Res Methodol 2017;17:139.

16 Denzin NK. Collecting and interpreting qualitative materials. Vol 3. Thousand Oaks, CA: SAGE, 2008.

17 Darawsheh W. Reflexivity in research: promoting rigour, reliability and validity in qualitative research. Int $J$ Ther Rehabil 2014;21:12:560-8.

18 Silverman D. Qualitative research. thousand oaks. CA: SAGE, 2016.

19 Meyerhardt JA, Niedzwiecki D, Hollis D. The content analysis guidebook. thousand oaks. CA: SAGE, 2016.

20 Cregan C. Can organizing work? an inductive analysis of individual attitudes toward Union membership. ILR Review 2005;58:282-304.

21 Piazza J, Ruby MB, Loughnan S, et al. Rationalizing meat consumption. The 4Ns. Appetite 2015;91:114-28.

22 Leroy F, Degreef F. Convenient meat and meat products. societal and technological issues. Appetite 2015;94:40-6.

23 Rong X, Peng Y, Yu H-P, et al. Cultural factors influencing dietary and fluid restriction behaviour: perceptions of older Chinese patients with heart failure. J Clin Nurs 2017;26:717-26.

24 Bell K. Cancer survivorship, mor(t)ality and lifestyle discourses on cancer prevention. Sociol Health IIIn 2010;32:349-64.

25 Wong-Kim E, Sun A, DeMattos MC. Assessing cancer beliefs in a Chinese immigrant community. Cancer Control 2003;10:22-8.

26 Shih FJ. Concepts related to Chinese patients' perceptions of health illness and person: issues of conceptual clarity. Accid Emerg Nurs 1996;4:208-15

27 Holm LE, Nordevang E, Ikkala E, et al. Dietary intervention as adjuvant therapy in breast cancer patients--a feasibility study. Breast Cancer Res Treat 1990;16:103-9.

28 Schwarzer R, Luszczynska AJEP. How to overcome healthcompromising behaviors: the health action process approach. Eur Psychol 2008;13:141-51. 